New (Oct 2019) Modifications

Delusion Formation as an Inevitable Consequence of a Radical Alteration in Lived Experience

Rachel Gunn \& Michael Larkin 


\title{
Delusion Formation as an Inevitable Consequence of a Radical Alteration in Lived Experience
}

\begin{abstract}
The definition of delusion is usually couched in terms of false beliefs held with conviction despite evidence to the contrary. Such beliefs are usually seen as impossible or implausible by others. In order to be classed as clinically significant, a delusion would lead a person to be distressed or to have significant problems with functioning. We note that a person ordinarily understands and negotiates the world based on familiar patterns derived from her cultural and historical experience. Thus, when her lived experience is altered, these assumptions about how the world works may be called in to question. Drawing on interviews conducted with people experiencing clinically significant delusions, we show how this alteration in lived experience manifests as emotional, affective and/or perceptual anomalies. We adopt the framework of the Enactive Approach which posits that a person interacts with her environment in terms of sense-making and we argue that radical alterations in lived experience can demand a renegotiating of how a person makes sense of this new world. We suggest that if the alteration in lived experience is sufficiently radical, then delusion formation is inevitable.
\end{abstract}

Keywords: Delusion, Affective, Emotional, Perceptual, Enactive Approach 


\section{Introduction}

People negotiate the world based on their experience of how their world works. A person is born into a family, the family consists of particular relationships and the family lives within a relatively circumscribed culture. A person might encounter or engage with other cultures or sub-cultures as she develops and finds her place in the world. Humans are flexible and adaptive. The difference between the lived experience of a hunter-gatherer in the Amazon Rainforest and an investment banker in the City of London highlights this flexibility. However, we can also imagine that there might be circumstances under which an alteration in a person's lived experience would be so radical that rapid adjustment is not possible (Parnas \& Handest, 2003). Faced with an unrecognisable world, a person might feel unable to cope with her lived experience. This might lead to despair, depression or even suicide. A person might require a new explanation of how the world works, in order to survive it.

In this paper, we draw upon some original research conducted with people who have experienced clinically significant delusions, in order to reflect upon these phenomena. We will argue that, in our participants' narrative accounts, a radical alteration in lived experience follows a trajectory that leads, via intense affective, perceptual and/or emotional experiences, to the forming of new explanations about the world. These new explanations may be difficult for others to comprehend, but they are not necessarily without meaning or internal coherence. Although it may seem to be a bold claim, the notion that we are sense-making beings embedded in environments over which we may have no control opens the possibility that any of us might need a delusional explanation to make sense of a radically altered world. That is not to say we imagine that all people would respond in the same way in extremis - simply that the (unique and complex) experiences each of us has gives us a (unique) 'breaking point.' We expand on the framework for this complexity further in what follows.

\subsection{Delusions and the Enactive Approach}

Many factors are implicated in shaping the development of human psychology. So when things go wrong and someone becomes mentally ill, there are many factors that might contribute to this (see Bentall, 2016 for an extensive list of candidates). People 
are dynamic and complex, and in most cases, so are the factors that causally contribute to their psychological distress.

The enactive approach posits that a vast array of factors (biological, psychological and environmental) are intermeshed to create a 'person-environment system'. This term invites us to notice that a person is not a discrete object; persons are comprised of bodies, stories, concepts, origins, commitments, connections, affordances - and so on - and are constantly reacting with their environment. Cognition emerges from the complex mereology of these many components. According to the enactive approach, a mind does not exist in isolation from a person or the world that the person inhabits. In the enactive approach, the mereology of the cognising (person-environment) system supposes that the parts (which include relational and environmental parts as well as bodily (person-level) parts) are arranged in a particular way, and that the relationship of the parts to each other is vital for the function of the whole. The function of the whole co-constitutive system is a property of the parts being arranged in this particular way. The enactive approach sees the person as primarily a sensemaking being and, as such, her primary goal is to make sense of her experience understood in terms of any situation or environment in which she finds herself (Varela, Rosch, \& Thompson, 1991).

\subsection{Aims of this Paper}

A person has desires and needs in relation to survival and procreation - and these all have affective components. A person cares about herself and how her needs are met. She makes decisions about action in the world based on her response to positivelyvalenced preferences and negatively-valenced objects, or situations to be avoided. The person's response is first bodily and then cognitive. A human being is (perhaps uniquely) able to respond to her situation at a number of different 'levels'. These levels might be described as somatic (or bodily), cognitive and meta-cognitive. For example a person might see a bear coming towards her and start to run away as a direct result of a somatic fear response (with no self-conscious thought about it at all). Alternatively, she might have a similar fear response but recognise it as such (through self-conscious cognition), and so weigh up the options and decide instead to hide rather than run. She might see a bear in a film, or safely contained at the zoo, and yet 
still experience a strong fear response, leading her to recognise that she has a fear of bears, and realise that this response is irrational (at a meta-cognitive level, through thinking about her thinking) and decide to do nothing. This affective content helps us to make sense of the world, and to identify those things which are experienced as positively and negatively valenced. In this paper, we will show and argue that a serious disruption in affective experience, arising from a person-environment system, may have an important role in the formation of delusions.

In section 2, we outline the methodology used for the empirical research, in section 3 we analyse the research findings and in section 4 we briefly discuss models of delusion, the implications of our findings and make some concluding remarks.

\section{Method}

\subsection{Approach}

The first author planned to interview, record and transcribe the experiences of people with active delusions, and then to analyse the transcripts using Interpretative Phenomenological Analysis (IPA). IPA has its origins in phenomenology and as such fits with an attempt to capture the nature of an experience for a given person in a given context. Smith and colleagues use Heidegger's notion of appearing and liken interpretation to a kind of detective work where the researcher is mining the material for possible meanings, thus allowing the phenomenon of interest to shine forth (Smith, Flowers, \& Larkin, 2009, p. 35). The double hermeneutic means that the researcher is always trying to make sense of the participant, who is in turn trying to make sense of what is happening in the context of her lifeworld as an embodied, situated person. As such, IPA is compatible with the enactive approach to cognition (Larkin, Eatough, \& Osborn, 2011). Using the double hermeneutic encourages a both/and approach on the part of the researcher: on the one hand to assume an empathic stance and imagine what it is like to be the participant and, at the same time, to be critical and probing in ways in which participants might be unwilling or unable to engage with themselves. IPA thus aims to produce rich experiential understandings of the phenomenon under investigation whilst remaining close to the participant's 
sense-making as well as developing a narrative of other possible meanings (Eatough \& Smith, 2008). This is in contrast to other qualitative methods such as ethnography, thematic analysis and grounded theory which do not specifically employ the double hermeneutic in this way (Howitt, 2016).

\subsection{Ethical review}

A detailed research protocol ${ }^{1}$ was written and submitted via the Integrated Research Application System (IRAS) for National Health Service (NHS) Ethical approval and for NHS Research and Development (R\&D) approval. The protocol was approved by the ethics committee and was approved by local (participating) NHS trusts.

\subsection{Sampling}

IPA requires in-depth data from relatively small samples of participants, due to its commitment to idiographic detail, and its sensitivity to context.

Our participants were sought via collaboration with local NHS mental health departments. Participants meeting the following criteria were interviewed:

- $\quad$ service users within local NHS mental health services

- having active delusion(s)

- able to give informed consent to participate in the study

- able to talk about their experiences

- at minimal risk (of distress) when talking about their experiences

- able to speak English (no translator is provided)

- willing to travel to a suitable NHS location to participate in the interviews

Four participants agreed to take part, and each was interviewed twice.

\footnotetext{
${ }^{1}$ IRAS research protocol number 167001
} 


\subsection{Data collection}

The first author conducted the interviews. Each participant was interviewed for approximately one hour on both occasions, using a semi-structured topic guide. In the first interview, the participants were asked about their history and the onset of their problems. In the second interview, participants were asked what their experience was like at the time of being interviewed. The focus was on what was important to the participant. The interviews were recorded, anonymised and transcribed.

\subsection{Data analysis}

Data analysis (analysis of each research participant's narrative) was undertaken using Interpretative Phenomenological Analysis (IPA). Narrative provides an important basis for the analysis of qualitative data and, whether we ask them to or not, participants in qualitative research interviews often shape their accounts as stories. Stories are not distractions from important information about experiences. Stories themselves are important information (Stephens \& Breheny, 2013).

IPA aims to understand what experience means to an individual, and also to identify themes across different people's experience. We wished to understand both the experience of the delusion and the meaning it held, as well as the context (in narrative terms) in which the delusion arose.

Using IPA, the transcripts were analysed recursively for each participant, and then across the different participants. Analysis in IPA follows a set of key principles (Smith et al., 2009), which are often implemented via a set of common processes: repeated listening, repeated reading of transcripts, reflection, detailed annotation and coding, clustering of themes, checking interpretations, re-checking and seeking credibility with a third party. We followed these generic processes, with the first author conducting the preliminary coding for experiential content (see Larkin \& Thompson, 2011 for coding strategies) and the second author providing reflection and credibility checking on the developing interpretations. IPA's processes are designed to ensure that interpretative claims are grounded in the data, rather than the 
researcher's biases and assumptions, and to produce an analytic account which is both meaningful and credible (Smith et al., 2009).

\section{Research Findings}

In the spirit of IPA's idiographic commitments, we begin by briefly describing the ordinary lived experience of each research participant, prior to the radical changes in their lived experiences in order to establish the context of our participants' accounts (section 3.1). We then present a more interpretative account in section 3.2.

\subsection{Context}

This qualitative research relies on the participant to tell his or her story in whatever way he or she chooses. An assumption that a person has told us what they deem to be significant is made here. Details about their lives before the onset of their delusions are limited, but it is possible to begin to understand what was important and meaningful to them and what they expected from life.

One of the research participants, Barbara, was married to a man who was a serial adulterer. He had already left or threatened to leave her on a number of occasions for other women. She had been desperate for him to stay with her and he had done so each time. Her marriage was extremely important to her and she had made decisions against her better judgement at her husband's behest in order to prevent him from leaving.

Another participant, Andrew, joined a 'job for life' workplace at a young age. He was the office junior and was required to respond to the demands of a number of different people. He was perhaps naïve and had little experience of relationships outside the family. He said a number of times while recounting his experiences, 'I didn't know how the world worked.' He bought a flat at the age of 21 and moved out from the parental home to live on his own. Shortly afterwards his father became ill and his mother became his father's carer. Andrew, who was very close to his parents, said he was 'left alone to my own devices.' He was focussed upon work and had a strong desire to do well. 
Alison, our third research participant, had two young children when her husband died by suicide. She worked as a legal secretary and had a keen interest in the law. She subsequently re-married and had two more children with her new husband. She continued to work because she enjoyed her job and she wanted to help support the family financially. She retired after working for over thirty years. Despite having some problems with her neighbours, she looked after some of them, and was involved with her community.

Caroline grew up in a family where she was required to do what her sister said or else she was punished. If she showed she was upset, her father would punish her. When she complained to her parents about her sister's behaviour they said they could not help her, because it was 'only your word against your sister's' (her sister was very careful not to get caught). Caroline started a relationship with a woman when she was in her teens and some time later they moved in to a house together. Caroline said that the relationship was good to start with. Caroline had a good job that she liked and was also training to qualify for a better job in her chosen field.

In the next section, we describe the ways in which their lived experiences were altered.

\subsection{Injustice, Unfairness and a Radical Alteration in Lived Experience}

\subsubsection{Barbara}

When Barbara's husband finally left her, she became extremely depressed:

I was really depressed, really down, really miserable. So I prayed as you do... I just want this pain to go away. So... um... I'd already been through a bad time, took an overdose, everything and then all of a sudden... I felt better after I'd prayed. (Barbara $1,1: 9-15)$

Barbara prays for resolution and for the pain to go away. The breakdown of her marriage represented a significant alteration in Barbara's lived experience. Her marriage gave her meaning and, although her husband was unreliable he had always returned to her in the past thus affording a form of stability. Guilt about the previous decisions she had made - which she had justified in order to keep her husband - 
subsequently came to the surface. Barbara experienced the injustice of this situation: she had compromised her principles to keep her husband, and then he had left her anyway.

I'd feel really really guilty, the guilt would like... eat you up like you've done murder or something. It was... it felt really really guilty." (Barbara 1, 3:36-38)

Two weeks later, she suddenly felt better. Her depression and mental anguish disappeared and she was enjoying life. Around this time, Barbara started to get the sense that she was being watched and that songs on the radio were presenting important messages to her. She did not seem distressed, worried or puzzled although she did think it was 'weird' and found it difficult to explain. She assumed there was an explanation; she just needed to work out what the explanation was. Then Barbara started seeing messages in the environment, for example, through road signs. Her attention was drawn to particular words. The amalgamation of these words gave her new messages. Again, she found this very difficult to explain. This is a common feature prior to delusion formation (Kapur, 2003).

It is unclear exactly when it started to happen, but at some point she experienced God replying to her questions and prayers. She said that God was talking to her directly 'by telepathy.' Barbara became overwhelmed by fear and guilt and the need to atone. She began to deny herself food and tried to give up smoking. Eventually she developed an elaborate schema where she was God's daughter and therefore did not have mortal needs (e.g. food), all other people were devils and were doomed to mortal lives, she could talk to God any time she wanted and he would talk back to her, God loved her unconditionally and forgave her all her sins, and she would live forever in Heaven:

He wouldn't let me do... he wouldn't let me do anything that would damage me... something that I wouldn't be able to live with. (Barbara 2)

I know I'm safe wherever I go. (Barbara 2) 
Barbara's new world-view enabled her to feel that she was loved and lovable, that she was intelligent, beautiful, important and was forgiven for past actions. This enabled her to make new meaning in this new world.

\subsubsection{Andrew}

Andrew described his workplace as a 'hellhole'. He found it very difficult to talk about this, resorting to analogy and generalisation:

It's that awful. You've seen the original 'Planet of the Apes'... film, 1964 I think it is with Charlton Heston... and you know how he's treated during it? ${ }^{2}$ Management treat you the... similar to that. That's how it felt. (Andrew 1)

He was utterly powerless in this situation. His whole idea of what the workplace should be like and how people should be treated is brought into question. He sounded contemptuous when he said:

So when... managers have more power than the worker they misuse it. It's human nature. Absolute power corrupts and absolute power corrupts absolutely. If you give people too much power they'll misuse it. (Andrew 1)

Andrew developed obsessional thoughts, checking behaviours, mild paranoia, had trouble sleeping and became addicted to sleeping tablets. He eventually sought help and was diagnosed with Obsessive Compulsive Disorder (OCD).

Andrew's new life represented a radical alteration in his lived experience. Prior to this he lived with his parents and enjoyed a relatively stress free life. He found that he was unable to negotiate relationships at work and felt huge anxiety in this regard. $\mathrm{He}$ became stressed and fearful. We assume he had not experienced injustice before; he found what he witnessed at work to be intolerable. Once the OCD took hold, he felt utter despair and 'torture.'

\footnotetext{
${ }^{2}$ In the film 'Planet of the Apes' human beings are treated like animals, used for slave labour, kept in cages and experiments are done on them.
} 
Andrew says he was taken over by God, wrote an email where he committed to righting some global wrongs, sent it to a large mailing list, called the police and called an ambulance. He said that God spoke to him:

...you need to take a massive leap of faith. Trust in me. Trust in God and send this email. You are the only person who can do it... And I literally wrote... everything... that it felt I was commanded to by God. (Andrew 2)

Andrew's delusion, that he was God's messenger and could mete out God's justice, allowed him to believe that God always had a plan for him. This gave meaning to the 'torture' that he had suffered and enabled resolution of the tension he felt about not being able to fully explain, understand or negotiate the world:

...even whilst all the suffering I'd been through, like during it, I'd always thought that this was potentially God's plan. For me. (Andrew 2)

This might help to restore his previously held ideas about the world being just. If there is no justice or fairness in the workplace, there is justice through God. Andrew, as God's messenger could commit to participating in making sure that justice was done by 'doing all that is necessary.'

\subsubsection{Alison}

Alison moved away from her anti-social neighbours and went to live near a family member. This relative was then falsely accused of sexually abusing his step-daughter. Alison attended court every day. She became stressed and started to have difficulty sleeping. He was found guilty of the crime and subsequently jailed. She described it as follows, and was clearly disdainful of the decision:

It was split.... and the judge said well I'm locking you up for the weekend... unless you can come back quickly with a ten two. So they did. And he was found guilty. He did from the May til Christmas and obviously he's got ten years now because you have ten years put on don't you, at home. He's done three years. But he didn't do it. (Alison 1)

\footnotetext{
${ }^{3}$ This is a quote from the film 'Batman Returns' - Andrew uses this quote a number of times and uses other 'hero' film analogies throughout the interviews.
} 
This constituted a radical alteration in Alison's lived experience. She previously had positive experiences with the law and the police (due to her job as a legal secretary and from personal experience) and she had faith in the police and the judicial system. She was horrified that, on this occasion, when her family member was relying on the law to prove that he was innocent the system has failed. She found this injustice difficult to understand.

Alison's world altered as the injustices accrued. She was falsely accused of crimes and reported to the police by a neighbour when she moved back to her home town. The harassment from her neighbour was so distressing that she decided to move house. Around this time she also found out she had a life-threatening heart condition. It is unclear exactly when this happened, but at some point Alison started to think that the neighbour, the local council and the police were working together to prove that she was guilty of something. The methods she described seemed implausible. When she moved house she found out that the old neighbour was friends with the new one. After this Alison went to bed and stayed there for four months only getting up to go to doctor's appointments and to see family.

Eventually she said she could hear other people having conversations (when those people were not present). She also said she could hear people who were present but who did not look like they were talking and could communicate with others without saying anything. She had difficulty explaining this and, at times, was adamant that this was happening and at other times she questioned the experience and wondered if it was 'all in her head':

I got the voices in my head... um... I felt that I could talk to people without moving my mouth, I could hear long distance conversations, but yeah sometimes I still do. And sometimes I walk past people and I feel as though I know what they're thinking. (Alison 1, 3:46-49)

Well, no, that is true. I could hear people's thoughts. And I used to go like this as I passed them [puts her fingers in her ears]. (Alison 2, 5:4-5)

If she was mis-identifying her own thoughts, fears, wishes and imaginings as external in some sense (either 'voiced' out loud or 'picked up' through telepathy) she required 
an explanation. If she thought these thoughts and voices were not her then she looked to the environment for an explanation. Those who were around her, such as neighbours and visiting police, seemed likely candidates. If she could pick up the thoughts of others then perhaps they could pick up her thoughts. This seemed to her to be true as what she experienced seemed like a conversation (using telepathy). Alison's new world incorporated these experiences. She did not need to worry about 'going mad' because she was telepathic. In this way she was able to have 'long distance conversations' with police and council officials in order to tell them her side of the story.

\subsubsection{Caroline}

Caroline's relationship, which started off well, began to break down. Her partner became verbally and physically aggressive. With hindsight Caroline could see that she was unable to acknowledge her own emotions or express them in a straightforward way. She was unaware of this at the time and she just put up with it while it got worse. She assumed that this abuse was 'normal' because she had experienced it from her sister and her father:

I never realised at the time I was in an abusive relationship... both mentally and physically.... Some people do it and some people don't and it was just normal to have people out there that did do them things..." (Caroline 1)

Her ability to negotiate the world became radically altered. She was used to being able to shut off her emotions and put up with anything. She now found she could no longer do this. She got physical symptoms and eventually had full blown panic attacks that she did not understand. She took a number of overdoses and ended up at the Accident and Emergency (A\&E) department of her local hospital. She could not say, at the time, why she was doing this. Her experience of herself as a robust person who could just get on with life was completely shattered. Caroline had, perhaps, experienced injustice all her life but because this was normalised she had developed coping strategies. She was re-assaulted by the injustice in her new relationship and found that her coping strategies no longer worked. 
Caroline developed a number of 'voices.' She described looking around for where the voices were coming from on some occasions, thinking there were speakers behind her somewhere, thinking there was a chip in her arm (put in by the government) and puzzling over how she was hearing what she was hearing. At the same time she also seemed to think it was normal in some sense.

She did not question the commanding nature of the voices. She simply incorporated them into her world as real, and felt compelled to do what they commanded. Sometimes she would resist, but the voice would persist and the threat would increase until she did what she was told. There are obvious parallels between this and her sister's behaviour ('do this or I'll hit you'), her father's behaviour ('don't get upset or I'll give you something to really be upset about') and her partner's abusive behaviour. This behaviour drew attention to her plight and mental health services became involved.

\section{Implications / discussion}

\subsection{Perceptual, Affective and Emotional Anomalies and a Radical Alteration in}

\section{Lived Experience.}

Barbara experienced being drawn towards things in the environment such as music on the radio, road signs and things that people were saying. Other things in the environment, such as certain people and places, repelled her and she had the sensation that she was being watched. Barbara felt despair about her husband leaving, guilt over past decisions and intense joy/love, fear and anger. At times these emotions felt objectless and they became attached to other experiences. Barbara needed an explanation for how she now experienced the world.

Alison found that she could 'hear' long distance conversations, read the minds of others and converse with people 'telepathically.' She at first found this peculiar and thought she might be ill. As this experience persisted she came to accept that something was happening to her that required explanation. 
Caroline was traumatised from an early age. Strong bodily feelings (such as panic) and a compulsion to behave in ways that she did not understand (e.g. taking overdoses) became part of Caroline's new world. She could not understand what was happening and sought an explanation.

Andrew was immersed in an unjust and frightening environment. He developed OCD and became obsessed with worrying about work and a training course he was doing. He then became overwhelmed by an inexplicable power and was compelled to write and send an email. He sought an explanation in order to make sense of his experiences.

All the research participants have experienced a radical alteration in lived experience characterised by affective, perceptual and emotional anomalies. These anomalies inform their lived experience and, as we are sense-making beings, demand an explanation. In each case the attempt at sense-making turns out to be delusional.

In what follows we look at models of delusions and how our findings might best be conceptualised.

\subsection{Some Models of Delusion}

Garety and Freeman's (2013) review of around 250 research papers on delusions suggests that stress, anxiety, worry and other emotions might be significant factors with regard to delusion formation and highlights the presence of perceptual or affective anomalies prior to the onset of delusion. They further suggest that it is important to conduct research to understand the factors that might be at play in relation to delusion formation and maintenance. They recognise the heterogeneity of delusions and questions the validity of diagnostic categories with regard to research and treatment, concluding that:

...the focus should be on understanding and treating the individual experiences that the patient is reporting, with the aim of reducing the distress and impact of each. In the clinic the experiences of the patient - rather than diagnosis - should be placed centre stage. (ibid) 
One assumption which underpins many psychological approaches to delusional beliefs is that delusions are formed as a result of understandable, but erroneous, interpretation. Across a range of conventional cognitive models, this error may be made due to biases arising from previous experiences, or deficits (e.g. in reality testing) arising from other aspects of psychotic syndromes (Barch, 2004). In Bayesian cognitive models, the error arises from a mismatch between the brain's predictions and subsequent perceptual information (Sterzer et al., 2018). At a conceptual level, these are not hugely dissimilar.

In contrast, the Enactive Approach offers us a different view. To be clear, we do not intend to claim that the enactive approach tells us exactly how or why a particular individual develops a delusion. Rather, the enactive approach gives a general framework that captures the complexity of individual experience, in the context of sense-making, and within a person-environment system. The Enactive view contains the idea that the 'error' of a delusional belief is understandable in terms of sensemaking within the complex mereology entailed through understanding cognition as an emergent property of a person-environment system. This means that the experience must be understood at the level of individual phenomenology in order to grasp the nature of the delusion. From a clinical perspective, we must therefore take seriously the environment in which a person finds herself as constitutive of delusion formation.

This difference in emphasis arises because the notion that a person is both embodied and embedded in the world is central to the Enactivist's conceptualisation of cognition (see Larkin, Eatough \& Osborn, 2011). A person cannot exist without a body. A person does not exist outside of, or separate from the environment in which she lives. She is embedded in the world in which she finds herself. Cognition is a property of the embodied embedded person and as such is subject to the complex mereology entailed in such a system (Varela et al., 1991). If mental activity is an emergent property of lived experience, then the biological, psychological and environmental are constitutive of cognition - and therefore they are also constitutive of phenomena such as delusions. 


\subsection{Limitations}

The category of 'radical alteration' is open and contains the kinds of alterations which are described by our participants in this study. We acknowledge that there is tension between describing participants' accounts and interpreting their meaning. In qualitative analysis, it is conventional for interpretative themes to be developed and conceptualised in the inductive manner which we have employed here, but this does not rule out the possibility of alternate interpretations. As a consequence, our claim is a deliberately cautious one relating to these four cases, and we hope that further work will be conducted to elaborate on the contents and boundaries of this category.

We have described the four cases in terms of sense-making and the enactive approach. Interpretation is a cornerstone of IPA and our overarching argument (that people are sense making beings who always strive to make sense of their world) is compatible with the Enactive Approach and with the data. There are, of course, other theoretical frameworks (including social constructivist, Kantian and many other approaches - see, for example, Georgaca, 2004; Radden, 2011; Kiran \& Chaudhury, 2009), but we have focused on the Enactive Approach for our argument because it seems to us to offer a particularly salient and helpful way of thinking about these data. We remain open to the possibilities which might be afforded by further dialogue with other models.

\subsection{Implications}

If delusions are understood as heterogeneous and as emerging from an understandable re-evaluation arising from a radical alteration in lived experience in the context of affective, emotional and perceptual anomalies, this demands an attempt to understand the totality of a person's experience. A person strives for sense-making in whatever environment she finds herself. From a clinical perspective, this demands an attempt to understand the phenomenology of the experience. There is a wide literature on the nature of stigma in mental illness (see for example Canadian Health Services Research Foundation, 2013; Mehta \& Farina, 1997) and framing delusion formation in this way helps us to reduce stigma: how can a person who is doing her best to make 
sense of her world be 'at fault' or 'bad'? It also helps us to take seriously the impact of environmental and affective factors: delusions are not simply 'faulty' or 'biased' thought processes. We can then recognise the complexity of the systems within which delusions arise. The complexity and individuality of human experience understood in terms of sense-making for each unique individual means that we move away from generalisations about how delusions are formed.

In this context the focus of treatment might then be directed towards affective, perceptual and emotional aspects of a person's lived experience as well as environmental and relational factors. To think of delusion simply in terms of 'false beliefs' which are 'firmly sustained despite... obvious proof or evidence to the contrary...' (American Psychiatric Association, 2013, p. 819) is to limit them to cognitive anomalies, over-simplify the experience and deny the meaning an experience might hold for a given individual.

\section{Acknowledgement (details tba - to preserve anonymity)}

One author (name to be advised) was supported by a European Research Council Consolidator Grant ('....' (to be advised); Grant Agreement .....) for Project '...' to be advised (PI: '...' to be advised).' 


\section{References}

American Psychiatric Association. (2013). Diagnostic and statistical manual of mental disorders : DSM-5. (5th ed.). Arlington, Va: American Psychiatric Association.

Barch, D. M. (2004). The Cognitive Neuroscience of Schizophrenia. Annual Review of Clinical Psychology, 1(1), 321-353. https://doi.org/10.1146/annurev.clinpsy.1.102803.143959

Bentall, R. (2016, February 19). All in the brain? Retrieved 20 April 2016, from https://blogs.canterbury.ac.uk/discursive/all-in-the-brain/

Canadian Health Services Research Foundation. (2013). Myth: reframing mental illness as a 'brain disease' reduces stigma. Journal of Health Services Research \& Policy, 18(3), 190-192. https://doi.org/10.1177/1355819613485853

Eatough, V., \& Smith, J. A. (2008). Interpretative Phenomenological Analysis. In C. Willig \& W. Stainton-Rogers, The SAGE Handbook of Qualitative Research in Psychology (pp. 179-194). 1 Oliver's Yard, 55 City

Road, London England EC1Y 1SP United Kingdom: SAGE Publications Ltd. https://doi.org/10.4135/9781848607927.n11

Georgaca, E. (2004). Factualization and Plausibility in Delusional Discourse.

Philosophy, Psychiatry, \&amp; Psychology, 11(1), 13-23.

https://doi.org/10.1353/ppp.2004.0037

Howitt, D. D. (2016). Introduction to Qualitative Research Methods in Psychology (3 edition). Harlow, United Kingdom: Pearson. 
Kapur, S. (2003). Psychosis as a State of Aberrant Salience: A Framework Linking Biology, Phenomenology, and Pharmacology in Schizophrenia. American Journal of Psychiatry, 160(1), 13-23. https://doi.org/10.1176/appi.ajp.160.1.13

Kiran, C., \& Chaudhury, S. (2009). Understanding delusions. Industrial Psychiatry Journal, 18(1), 3-18. https://doi.org/10.4103/0972-6748.57851

Larkin, M., Eatough, V., \& Osborn, M. (2011). Interpretative phenomenological analysis and embodied, active, situated cognition. Theory \& Psychology, $21(3), 318-337$.

Larkin, M., \& Thompson, A. R. (2011). Interpretative Phenomenological Analysis in Mental Health and Psychotherapy Research. In D. Harper \& A. R. Thompson (Eds.), Qualitative Research Methods in Mental Health and Psychotherapy: A Guide for Students and Practitioners (pp. 101-116). Chichester, West Sussex: Wiley-Blackwell.

Mehta, S., \& Farina, A. (1997). Is Being 'Sick' Really Better? Effect of the Disease View of Mental Disorder on Stigma. Journal of Social and Clinical Psychology; New York, 16(4), 405-419.

Parnas, J., \& Handest, P. (2003). Phenomenology of anomalous self-experience in early schizophrenia. Comprehensive Psychiatry, 44(2), 121-134. https://doi.org/10.1053/comp.2003.50017

Radden, J. (2011). On Delusion. London: Routledge.

Smith, J. A., Flowers, P., \& Larkin, M. (2009). Interpretative phenomenological analysis: theory, method and research. Los Angeles: SAGE. 
Stephens, C., \& Breheny, M. (2013). Narrative Analysis in Psychological Research: An Integrated Approach to Interpreting Stories. Qualitative Research in Psychology, 10(1), 14-27. https://doi.org/10.1080/14780887.2011.586103

Sterzer, P., Adams, R. A., Fletcher, P., Frith, C., Lawrie, S. M., Muckli, L., ... Corlett, P. R. (2018). The Predictive Coding Account of Psychosis. Biological Psychiatry, 84(9), 634-643. https://doi.org/10.1016/j.biopsych.2018.05.015

Varela, F. J., Rosch, E., \& Thompson, E. (1991). The embodied mind : cognitive science and human experience. Cambridge, Mass.: MIT Press. 